\title{
Características do sêmen e maturidade sexual de touros jovens da raça Tabapuã (Bos taurus indicus) em diferentes manejos alimentares
}

\author{
[Semen characteristics and sexual maturity of young Tabapuã (Bos taurus indicus) bulls under different feeding \\ management]
}

\author{
A.B. Corrêa, V.R. Vale Filho*, G.S.S. Corrêa, V.J. Andrade, M.A. Silva, J.C. Dias \\ Escola de Veterinária - UFMG \\ Caixa Postal 567 \\ 30123-970 - Belo Horizonte, MG
}

\begin{abstract}
RESUMO
Estudaram-se as variações no peso corporal, circunferência escrotal, características do sêmen e classificação andrológica por pontos (CAP) em 516 touros jovens da raça Tabapuã, de um (12 a 17 meses) e dois anos (21 a 27 meses) de idade, criados sob três regimes alimentares. Os regimes alimentares foram os seguintes: regime 1: animais criados em pasto, com suplementação mineral; regime 2: animais criados em pasto com suplementação de cana-de-açúcar, uréia $(0,5 \%)$ e $2 \mathrm{~kg} /$ animal/dia de concentrado comercial ( $84 \% \mathrm{NDT}$ e $18 \% \mathrm{~PB})$ no período da seca (julho a setembro); regime 3: animais estabulados que receberam, a partir do desmame (maio), dieta balanceada composta por cana-de-açúcar, capim-elefante, uréia $(0,5 \%)$ e concentrado comercial $(88 \% \mathrm{NDT}$ e $19 \% \mathrm{~PB})$, que variou de $2 \mathrm{~kg} / \mathrm{animal} /$ dia até atingir $6 \mathrm{~kg} /$ animal/dia na idade adulta (27 meses de idade). Animais de um ano de idade submetidos ao regime alimentar 3 foram mais pesados $(\mathrm{PC}=416 \mathrm{~kg})$ e apresentaram maiores circunferência escrotal $(\mathrm{CE}=29,6 \mathrm{~cm})$, volume seminal $(5,7 \mathrm{ml})$ e motilidade espermática $(42 \%)$, que os animais dos regimes 1 e 2 . Não houve diferença entre as médias de peso corporal 232,44 e 279,34kg, circunferência escrotal 20,02 e $22,16 \mathrm{~cm}$, volume seminal 2 e $2,12 \mathrm{ml}$ e motilidade espermática 14,17 e $20,27 \%$, dos animais nos regimes alimentares 1 e 2, respectivamente. Os animais de dois anos de idade, sob regime 3, apresentaram maior PC $(490,82 \mathrm{~kg}), \mathrm{CE}(33,56 \mathrm{~cm})$ e CAP $(71,71)$, em comparação aos animais dos regimes 1 e 2 que apresentaram PC $(378,91$ e $409,2 \mathrm{~kg})$, CE $(30,66$ e $30,31 \mathrm{~cm})$ e CAP $(63,55$ e 66,05$)$, respectivamente. Houve diferença entre os animais do regime 3 e os animais dos regimes 1 e 2, para PC, CE e volume seminal. Animais maturos, sob regime 3, apresentaram 497,15kg de PC, 33,81 cm de CE e 4,83 $\mathrm{ml} \mathrm{de}$ volume seminal, enquanto os animais do R1 apresentaram 388,53kg de PC, 30,81 cm de CE e 2,92 $\mathrm{ml} \mathrm{de}$ volume, cujas médias não diferiram estatisticamente das apresentadas pelos animais do regime 2, que foram: $412,32 \mathrm{~kg}$ para $\mathrm{PC}, 31,56 \mathrm{~cm}$ para $\mathrm{CE}$ e $3,93 \mathrm{ml}$ para volume seminal. Touros imaturos aos dois anos de idade apresentaram maior PC $(479,22 \mathrm{~kg})$ e CE $(32,96 \mathrm{~cm})$ no regime 3 , que os PC e CE dos animais dos regimes $1(367,36 \mathrm{~kg}$ e $29,56 \mathrm{~cm})$ e $2(402,69 \mathrm{~kg}$ e $29,55 \mathrm{~cm})$. A suplementação alimentar interfere no desempenho reprodutivo, com efeitos positivos sobre peso corporal, circunferência escrotal, características físicas do sêmen e CAP de touros jovens Tabapuã.
\end{abstract}

Palavras-chave: bovino, Tabapuã, característica seminal, regime alimentar, touros jovens, desenvolvimento sexual

\begin{abstract}
Body weight (BW), scrotal circumference (SC), semen characteristics and Breeding Soundness Evaluation-Zebu (BSE-Z) of 516 young Tabapuã breed bulls, raised under different feeding regimen, were evaluated for one year old bulls (12-17 months of age) and two year old bulls (21-27 month of age). The feed regimens were the following: feeding regimen 1 - animals raised under pasture condition plus mineral supplementation, feeding regimen 2 - animals raised under pasture condition and supplemented
\end{abstract}

Recebido em 2 de maio de 2005

Aceito em 31 de março de 2006

E-mail: andrebritocorrea@yahoo.com.br 


\section{Corrêa et al.}

during the dry season with sugar cane and urea (0.5\%) plus $2 \mathrm{~kg} /$ animal/day of a commercial concentrate (84\%NDT and $18 \%$ crude protein) fed twice a day, and feeding regimen 3 -feed lot animals fed balanced diets with the following composition: sugar cane, elephantgrass, urea $(0.5 \%)$ and commercial concentrate (88\%NDT and $19 \%$ crude protein) varying from $2 \mathrm{~kg} /$ animal/day for young animals to $6 \mathrm{~kg} /$ animal/day at adult age (27 months), fed three times a day. One year old animals on feeding regimen 3 were heavier $(B W=416 \mathrm{~kg})$, and had higher $(P<0,05)$ scrotal circumference $(29.6 \mathrm{~cm})$, semen volume $(5.7 \mathrm{ml})$ and spermatic motility (42\%) than animals on feed regimens 1 and 2. No difference between animals on feeding regimens 1 and 2 were observed for $B W(232.44$ and $279.34 \mathrm{~kg})$, scrotal circumference $(20.02 \mathrm{~cm}$ and $22.16 \mathrm{~cm})$, semen volume (2 and $2.12 \mathrm{ml})$, and spermatic motility $(14.17 \%$ and 20.27\%), respectively. Two years old animals on feeding regimen 3 were heavier ( $B W=490.82 \mathrm{~kg})$, and had higher scrotal circumference $(S C=33.56 \mathrm{~cm})$ and andrologic classification $(A C P=71.71)$ than animals on feeding regimens 1 and 2, which showed, $B W(378.91$ and $409.2 \mathrm{~kg}), S C(30.66$ and $30.31 \mathrm{~cm})$ and $A C P$ (63.55 and 66.05), respectively. Difference between animals on feeding regimen 3 and animals on regimens 1 and 2 were observed for $B W, S C$ and $S V$. Mature animals showed $B W=497.5 \mathrm{~kg}$, $S C=33.81 \mathrm{~cm}$ and $S V=4,83 \mathrm{ml}$. Animals on feeding regimen $1(B W=388.53 \mathrm{~kg}, S C=30.81 \mathrm{~cm}$ and $S V=2.92 \mathrm{ml})$, were not statistically different from animals on feeding regimen 2 (BW=412.32kg, $S C=31.56 \mathrm{~cm}$ and $S V=3.93 \mathrm{ml})$. Immature animals were heavier $(B W=479.22 \mathrm{~kg})$ and had higher $S C$ $(S C=32.96 \mathrm{~cm})$ on feeding regimen 3 , than animals on feeding regimen $1(B W=367.36$ and $S C=29,56 \mathrm{~cm})$, and animals on feeding regimen $2(B W=402.69 \mathrm{~kg}$ and $S C=29.55 \mathrm{~cm})$. Feed supplementation influences reproductive performance, and shows positive effect on body weight, scrotal circumference, physicals characteristics of semen and BSE of young Tabapuã bulls.

Keywords: bull, feeding regimen, seminal characteristic, Tabapuã, young bull, sexual development

\section{INTRODUÇÃO}

Vários trabalhos têm relatado a fertilidade como a primeira prioridade econômica em gado de corte, seguida pelas taxas de crescimento e qualidade de carcaça (Bergmann, 1993). Na seleção de reprodutores, além das características zootécnicas fenotípicas, tais como peso corporal (numa determinada idade), desenvolvimento ponderal, qualidade de carcaça e tipo racial, deve-se dar ênfase à saúde reprodutiva dos animais, com avaliações clínicas que mostrem a normalidade dos órgãos reprodutivos e se há habilidade sexual, pela capacidade quantitativa e qualitativa da produção de espermatozóides aptos à fertilização.

Existem evidências de que $5 \%$ dos touros em uso, em monta natural, são estéreis e que mais de $30 \%$ são subférteis. Como o sistema de produção brasileiro caracteriza-se basicamente por criação extensiva, em que $95 \%$ da reprodução é realizada por monta natural, a campo, e apenas $5 \%$ por inseminação artificial, pode-se perceber a importância do touro no sistema de produção (Dode, 1997).

Apesar da grande potencialidade dos animais de raças zebuínas para a reprodução, estudos têm mostrado que limitações do meio ambiente podem atrasar a puberdade e a maturidade sexual, principalmente em animais geneticamente superiores, os quais têm demanda nutricional maior (Tegegne et al., 1992b; Rekwot et al., 1997b; Rodriguez-Urbina et al., 2002).

Apesar de ainda pouco estudada, a raça Tabapuã vem se destacando na bovinocultura de corte do Brasil graças à sua precocidade, fertilidade, conformação frigorífica e alta complementaridade em cruzamento com outras raças, especialmente a Nelore, para diversas características zootécnicas (Vale Filho et al., 2003).

No presente trabalho, objetivou-se estudar as variações na circunferência escrotal e nas características do sêmen de tourinhos Tabapuã, de um e dois anos de idade, criados em três regimes alimentares.

\section{MATERIAL E MÉTODOS}

Avaliaram-se 516 animais da raça Tabapuã, os quais foram agrupados em duas idades: um ano (12 a 17 meses), com 246 animais, e dois anos (21 a 27 meses), com 270 animais. 
$\mathrm{Na}$ fase de cria, antes da desmama, os animais, além do leite materno, receberam suplementação mineral enriquecida com fontes protéicas e energéticas em sistema creep-feeding até dois meses de vida. Ao atingirem sete meses de idade, foram desmamados e separados ao acaso, formando-se três grupos distintos, cada um submetido a regime alimentar diferente. Os grupos criados em pasto foram manejados em piquetes de Brachiaria brizantha de boa qualidade, recebendo suplemento mineral completo à vontade.

No regime 1 (R1), os animais foram criados e recriados em pasto, apenas com suplementação mineral; no regime 2 (R2), os animais foram criados em pasto, receberam suplementação alimentar de cana-de-açúcar (Saccharum officinarum), uréia e $2 \mathrm{~kg} / \mathrm{animal} / \mathrm{dia}$ de concentrado comercial $(84 \% \mathrm{NDT}$ e $18 \% \mathrm{~PB})$, no período da seca (julho a setembro), em dois tratos diários; e no regime 3 (R3), os animais permaneceram estabulados após desmame, receberam dieta balanceada, composta por volumoso de cana-de-açúcar, capim-elefante (Pennisetum purpureum), uréia $(0,5 \%)$ e concentrado comercial (88\%NDT e 19\%PB), começando com $2 \mathrm{~kg} /$ animal $/$ dia até atingirem $6 \mathrm{~kg} /$ animal $/$ dia na idade adulta $(27$ meses de idade), dividida em três tratos diários. Para o cálculo do peso corporal (PC), em cada regime alimentar, os animais de um e dois anos foram pesados individualmente.

A circunferência escrotal (CE) foi medida por meio de fita métrica flexível, na posição mediana do escroto, no ponto de maior diâmetro da bolsa escrotal. Para a coleta de sêmen, adotou-se o método da eletroejaculação. Imediatamente após a coleta, realizou-se a avaliação do volume (Vol), turbilhonamento (Turb), motilidade (Mot), vigor e concentração espermática (Conc), e parte do sêmen foi acondicionado em $1 \mathrm{ml}$ de solução formol-salina tamponada Hancock (1957) e levada ao laboratório para estudo da morfologia dos espermatozóides ejaculados, os quais foram classificados de acordo com as anormalidades encontradas, em: defeitos maiores (DM), defeitos menores (Dm) e defeitos totais (DT), segundo a metodologia descrita por Blom (1973).

As avaliações da Mot, Turb e Vigor dos espermatozóides foram realizadas segundo Manual... (1998). Para a concentração espermática, seguiu-se a recomendação de Hancock (1957) e, para o estudo da morfologia, usou-se o critério de Blom (1973).

O sêmen dos touros com dois anos de idade, classificados como andrologicamente normais, foram submetidos à classificação andrológica por pontos (CAP), segundo metodologia descrita por Vale Filho (1989) e Vale Filho et al. (2001). Adotou-se a classificação de animais sexualmente maturos para aqueles que se apresentavam com defeitos maiores inferiores a $15 \%$ e defeitos espermáticos totais inferiores a $30 \%$ (Vale Filho et al., 1989). Os animais sexualmente imaturos foram os que não se enquadraram nesta classificação.

Realizou-se análise de variância, utilizando o SAEG (Sistemas..., 2004), para PC, idade, CE, Vol, Turb, Mot, Vigor, Conc, DM, Dm, DT e o CAP. Para as comparações entre os animais sexualmente maturos e imaturos, foi utilizado o teste qui-quadrado, com nível de significância de $5 \%$ de probabilidade.

\section{RESULTADOS E DISCUSSÃO}

$\mathrm{Na}$ Tab. 1, estão as médias de PC, CE, características do sêmen (Vol, Turb, Mot, Vigor, Conc, DM, Dm e DT dos espermatozóides ejaculados) em touros Tabapuã com um ano de idade (12 a 17 meses), segundo os regimes alimentares.

Foram observadas diferenças significativas entre os regimes alimentares em animais com um ano de idade $(\mathrm{P}<0,05)$ para as variáveis $\mathrm{PC}, \mathrm{CE} \mathrm{e}$ Mot, o que demonstra a influência do regime nutricional sobre as variáveis estudadas (Tab. 1). Os animais do R3 apresentaram melhores resultados para o PC e CE que os animais dos demais regimes. Entretanto, animais do R2 apresentaram melhores resultados do que os do R1. Dias et al. (2003), ao trabalharem com machos da raça Guzerá, também observaram efeito do regime alimentar sobre o PC, CE e Mot, com melhores resultados para os animais estabulados. As variáveis PC e CE apresentaram o mesmo comportamento por serem relacionadas entre si nessa idade, isto é, os de maior PC apresentam as maiores CE. Resultados semelhantes foram observados por Valvasori et al. (1985); Silva et al. (1991); Unanian et al. 
(2000), que registraram associação entre desenvolvimento testicular e ponderal em bovinos.

Fonseca et al. (1997) e Fonseca (2000) sugerem a classificação andrológica de touros zebus com base na circunferência escrotal e nas características morfofisiológicas do sêmen. Segundo esses autores, animais na faixa etária de 12 a 18 meses e com CE maior que $26 \mathrm{~cm}$, seriam classificados como excelentes. Pode-se observar que, neste trabalho, os animais sob regime R3 apresentaram $\mathrm{CE}$ maior que $29 \mathrm{~cm}$ e, sob os demais regimes, menores que $23 \mathrm{~cm}$ (Tab. 1). Isso evidencia a importância do regime nutricional na expressão gênica dos animais com maiores potenciais, de forma a auxiliar na identificação dos animais geneticamente superiores.

Para as variáveis Vol e Mot, também foram observados melhores resultados em animais do R3 $(\mathrm{P}>0,05)$ em relação aos outros grupos estudados, bem como entre os regimes 1 e 2 entre si. Estes resultados sustentam a hipótese de que os animais com dieta suplementar têm melhor condição de expressar seu potencial genético. A diferença de PC nessa idade reforça a idéia de que o potencial reprodutivo de touros jovens pode ser influenciado por deficiências nutricionais. Alimentação deficiente influencia o desenvolvimento corporal e testicular de touros e provoca atraso no aparecimento da puberdade, sendo conseqüência do menor desenvolvimento dos testículos e demais órgãos genitais (Tegegne et al., 1992a).

Não foram observadas diferenças $(\mathrm{P}>0,05)$ entre os regimes alimentares para Turb, Vigor, Conc, DM, Dm e DT nos espermatozóides ejaculados. A explicação para o aumento $(\mathrm{P}<0,05)$ da $\mathrm{CE}$ diferenciado nos três regimes alimentares (R1, R2 e R3), mas sem reflexo diretamente significativo $(\mathrm{P}>0,05)$ sobre as características morfológicas dos espermatozóides ejaculados (DM, Dm, DT) na idade de um ano (Tab. 1), para tourinhos Bos taurus indicus, tem relação com o aparecimento inicialmente nos túbulos seminíferos da puberdade, somente de divisões mitóticas, nas células gametogênicas (2n cromossomos), de espermatogônias até espermatócitos primários, havendo, como conseqüência, aumento do diâmetro dos túbulos seminíferos e, também, na $\mathrm{CE}(\mathrm{P}<0,05)$.
Numa segunda fase, progressivamente, ocorrem as divisões meióticas ( $2 \mathrm{n}$, para $\mathrm{n}$ cromossomos), com o aparecimento dos espermatócitos secundários, que evoluem até os primeiros espermatozóides no ejaculado. Assim, não há, na primeira fase de um ano de idade, relação direta entre aumento $(\mathrm{P}<0,05)$ da $\mathrm{CE}$ e as modificações $(\mathrm{P}>0,05)$ da Conc, DM, DT, nos espermatozóides ejaculados. Numa primeira fase da puberdade, os testículos crescem (CE) e, somente numa segunda fase, os espermatozóides aparecem no ejaculado, inicialmente com elevada proporção de DM e DT, que diminuem com a chegada da maturidade sexual, conforme relatado por Vale Filho (1986).

A Conc $\left(\times 10^{6} / \mathrm{ml}\right)$ tem relação direta com o número de espermatozóides no ejaculado e o volume do líquido seminal presente no sêmen, que vem principalmente das vesículas seminais.

Em animais Bos taurus indicus puberais (um ano de idade), a Conc está ainda baixa em razão do número reduzido de espermatozóides produzidos e ejaculados, sendo dependente da relação mitose-meiose, inicialmente observada nos túbulos seminíferos, que nesse período ainda estão em fase de crescimento e de maturação sexual (Vale Filho et al., 2001).

Os animais do R3 apresentaram maior Vol $(\mathrm{P}<0,05)$ que os dos outros dois regimes estudados, isso indica que o desenvolvimento das glândulas anexas ocorreu mais precocemente no $\mathrm{R} 3$, principalmente das vesículas seminais.

Conforme observado por Vale Filho (1989), o Turb forte no sêmen do touro somente é observado quando existem altos e simultâneos valores de Conc, Mot e Vigor, no ejaculado, e baixos índices de DM, Dm e DT. No presente experimento, os animais do R3 mostraram-se com índices mais elevados de Conc, Mot e Vigor $(\mathrm{P}<0,05)$, e DM, Dm e DT mais baixos, que os dos R2 e R1.

O método da eletroejaculação para a coleta de sêmen no touro é considerado o mais prático e de melhor eficiência para animais jovens. Quando aplicado corretamente, leva ao condicionamento coletivo de todos os indivíduos que, em geral, respondem bem ao estímulo elétrico, na inervação parassimpática, via retal. $\mathrm{Na}$ primeira fase de excitação, são aplicados de cinco a seis 
estímulos intermitentes com igual amplitude $(0,50 \mathrm{~A})$, até que o líquido seminal apareça no copo coletor (límpido e translúcido), o que ocorre de 10 a 20 segundos. Na segunda fase de estímulos, que ocorre de 21 a 40 segundos após a primeira, surge turvação no líquido seminal, ligeiramente opaca, o que indica presença de espermatozóides na ejaculação.

Nesse ponto, mais dois estímulos são processados, com ligeira e maior persistência do estímulo elétrico e menor amplitude de amperagem $(0,30-0,50)$ com duração de 30 a 40 segundos. Dependendo da reação individual, a ejaculação se processa e há total turvação no líquido. Nessa seqüência, a coleta do sêmen, após os estímulos elétricos, nunca ultrapassa de 60 a 80 segundos em touros com um ano de idade, conforme já foi observado por Vale Filho (1989) e Vale Filho et al., (2001).

O método é simples, e a resposta ao estímulo elétrico, para obter completa ejaculação, foi semelhante para todos os tourinhos, que reagiram da mesma forma. Não foi observado qualquer transtorno individual, em qualquer coleta, que pudesse mascarar os resultados.

Tabela 1. Médias de peso corporal (PC), circunferência escrotal (CE) e características do sêmen em 246 touros Tabapuã com um ano de idade, de acordo com o regime alimentar

\begin{tabular}{cccccccccccc}
\hline $\begin{array}{c}\text { Regime } \\
\text { alimentar }\end{array}$ & $\begin{array}{c}\mathrm{PC} \\
(\mathrm{kg})\end{array}$ & $\begin{array}{c}\mathrm{CE} \\
(\mathrm{cm})\end{array}$ & $\begin{array}{c}\text { Vol } \\
(\mathrm{ml})\end{array}$ & $\begin{array}{c}\text { Conc } \\
\left(\mathrm{x} 10^{6} / \mathrm{ml}\right)\end{array}$ & $\begin{array}{c}\text { Turb } \\
(1-5)\end{array}$ & $\begin{array}{c}\text { Mot } \\
(\%)\end{array}$ & $\begin{array}{c}\text { Vigor } \\
(1-5)\end{array}$ & $\begin{array}{c}\text { DM } \\
(\%)\end{array}$ & $\begin{array}{c}\text { Dm } \\
(\%)\end{array}$ & $\begin{array}{c}\text { DT } \\
(\%)\end{array}$ \\
\hline 1 & $232,44 \mathrm{c}$ & $20,02 \mathrm{c}$ & $2,00 \mathrm{~b}$ & 51,83 & 1,00 & $14,17 \mathrm{~b}$ & 3,00 & 55,83 & 4,17 & 60,00 \\
2 & $279,34 \mathrm{~b}$ & $22,16 \mathrm{~b}$ & $2,12 \mathrm{~b}$ & 42,95 & 1,14 & $20,27 \mathrm{~b}$ & 3,17 & 62,82 & 5,28 & 68,17 \\
3 & $416,00 \mathrm{a}$ & $29,60 \mathrm{a}$ & $5,70 \mathrm{a}$ & 55,40 & 1,40 & $42,00 \mathrm{a}$ & 4,20 & 46,25 & 10,00 & 56,25 \\
\hline
\end{tabular}

Valores seguidos de letras distintas na coluna diferem entre si pelo teste Student-Newman-Keuls (P<0,05).

Vol=volume seminal, Turb=turbilhonamento, Mot=motilidade, Conc=concentração espermática, $\mathrm{DM}=\mathrm{defeitos}$ maiores,

$\mathrm{Dm}=$ defeitos menores, $\mathrm{DT}=$ defeitos totais

$1=$ somente em pasto; $2=$ pasto+suplementação na seca; $3=$ =stabulado.

$\mathrm{Na}$ Tab. 2, são apresentadas as médias de PC, $\mathrm{CE}$, características do sêmen e CAP para os três regimes alimentares, em tourinhos da raça Tabapuã com dois anos de idade. Houve efeito de o regime alimentar $(\mathrm{P}<0,05)$ para as variáveis PC, CE, Vol, Turb e CAP. Os animais do R3 foram mais pesados do que os do R2, e estes mais do que os do R1, o que evidencia a importância da alimentação suplementar estratégica.

Os melhores resultados para CE e CAP foram encontrados para animais do R3, e não houve diferença $(\mathrm{P}>0,05)$ entre os animais do $\mathrm{R} 1$ e $\mathrm{R} 2$. Como o CAP é índice de classificação dos touros aptos à reprodução, foi observado que o manejo alimentar aumentou o CAP. Animais com maiores CAP(s) podem ser acasalados com maior número de vacas por estação de monta. Isso pode resultar em maior economia porque a utilização de menor número de touros resulta em menor custo por bezerro desmamado, o que foi também observado por Fonseca (2000) e Vale Filho (2001). Ressalta-se que os animais do R1 e do R2 também foram considerados aptos para a reprodução, pois apresentaram $\mathrm{CAP}>60$ pontos (Vale Filho, 1989; Vale Filho et al., 2001). Os três regimes alimentares, portanto, não causaram deficiência nutricional que pudesse influir no desempenho reprodutivo dos touros. RodriguesUrbina et al. (2002) observaram maior PC $(416,6 \mathrm{~kg})$ e maior CE $(33,6 \mathrm{~cm})$ em animais Brahman, suplementados e com 24 meses de idade, em comparação com animais criados somente em pasto, os quais apresentaram PC de $372,3 \mathrm{~kg}$ e $\mathrm{CE}$ de $31,1 \mathrm{~cm}$.

Não houve diferença de Vol e Turb entre os animais dos regimes $\mathrm{R} 2$ e R3, que apresentaram resultados melhores do que os animais do $\mathrm{R} 1$. Isso significa que os animais do $\mathrm{R} 2$ receberam alimentação capaz de atender seus requerimentos nutricionais para expressão destas características.

Houve diferença de Vol e Turb entre animais dos regimes $\mathrm{R} 3$ e $\mathrm{R} 2$ em relação aos do $\mathrm{R} 1$, porém não houve diferença de Conc $\left(\times 10^{6} / \mathrm{ml}\right)$ entre os três regimes, em razão de os dados terem sido expressos na base de $\mathrm{ml}$. Animais dos regimes R3 e R2 apresentaram Vol 2/3 maior que os animais do R1, porém, em decorrência do maior Vol de ejaculado, a Conc espermática permaneceu semelhante. 


\section{Corrêa et al.}

Tabela 2. Médias de peso corporal (PC), circunferência escrotal (CE), características do sêmen e classificação andrológica por pontos (CAP) em 270 touros da raça Tabapuã com dois anos de idade, de acordo com o regime alimentar

\begin{tabular}{cccccccccccc}
\hline $\begin{array}{c}\text { Regime } \\
\text { alimentar }\end{array}$ & $\begin{array}{c}\mathrm{PC} \\
(\mathrm{kg})\end{array}$ & $\begin{array}{c}\mathrm{CE} \\
(\mathrm{cm})\end{array}$ & $\begin{array}{c}\text { Vol } \\
(\mathrm{ml})\end{array}$ & $\begin{array}{c}\text { Conc } \\
\left(\times 10^{6} / \mathrm{ml}\right)\end{array}$ & $\begin{array}{c}\text { Turb } \\
(1-5)\end{array}$ & $\begin{array}{c}\text { Mot } \\
(\%)\end{array}$ & $\begin{array}{c}\text { Vigor } \\
(1-5)\end{array}$ & $\begin{array}{c}\text { DM } \\
(\%)\end{array}$ & $\begin{array}{c}\text { Dm } \\
(\%)\end{array}$ & $\begin{array}{c}\text { DT } \\
(\%)\end{array}$ & $\begin{array}{c}\text { CAP } \\
(0-100)\end{array}$ \\
\hline 1 & $378,91 \mathrm{c}$ & $30,66 \mathrm{~b}$ & $2,82 \mathrm{~b}$ & 324,74 & $1,68 \mathrm{~b}$ & 48,08 & 4,78 & 21,93 & 6,39 & 27,94 & $63,55 \mathrm{~b}$ \\
2 & $409,20 \mathrm{~b}$ & $30,31 \mathrm{~b}$ & $3,90 \mathrm{a}$ & 322,11 & $2,36 \mathrm{a}$ & 53,42 & 4,65 & 20,44 & 6,99 & 27,44 & $66,05 \mathrm{~b}$ \\
3 & $490,82 \mathrm{a}$ & $33,56 \mathrm{a}$ & $4,36 \mathrm{a}$ & 364,44 & $2,20 \mathrm{a}$ & 51,17 & 4,67 & 16,27 & 6,03 & 22,30 & $71,71 \mathrm{a}$ \\
\hline
\end{tabular}

Valores seguidos de letras distintas na coluna diferem entre si pelo teste Student-Newman-Keuls $(\mathrm{P}<0,05)$.

$\mathrm{Vol}=$ volume seminal, Turb=turbilhonamento, Mot=motilidade, Conc=concentração espermática, $\mathrm{DM}=\mathrm{defeitos}$ maiores, $\mathrm{Dm}=$ defeitos menores, $\mathrm{DT}=$ defeitos totais, $\mathrm{CAP}=$ classificação andrológica por pontos (Vale Filho, 1989)

$1=$ somente em pasto; 2 =pasto+suplementação na seca; $3=$ =stabulado.

Aos dois anos de idade, houve diferença no CAP de animais do R3 em relação aos dos regimes R2 e R1 $(\mathrm{P}<0,05)$, apesar de os animais dos três regimes terem apresentado $\mathrm{CAP}>60$ o que, de acordo com Vale Filho (1989), capacita andrologicamente touros para a reprodução.

Na Tab. 3, estão apresentadas as médias de PC, $\mathrm{CE}$, características seminais e CAP dos animais provenientes dos três regimes alimentares e classificados como sexualmente maturos, de acordo com Vale Filho et al., (1989). Na Tab. 4, estão, à exceção do CAP, os valores referentes às variáveis dos animais classificados como sexualmente imaturos, de acordo com Vale Filho et al. (1989). O CAP é índice recomendado somente para a classificação de touros sem distúrbios andrológicos (com gametogênese normal).

Nos animais sexualmente maturos, com gametogênese e demais funções reprodutivas normais, $\mathrm{DM}<15 \%$ e $\mathrm{DT}<30 \%$, observou-se diferença $(\mathrm{P}<0,05)$ entre regimes alimentares para PC, CE e Vol, sendo registrados melhores resultados na seguinte seqüência R3, R2 e R1 (Tab. 3).
Não foi observada diferença $(\mathrm{P}>0,05)$ entre regimes alimentares para as demais variáveis Turb, Mot, Vigor, Conc, DM, Dm, DT e CAP. Isso sugere que, aos dois anos de idade, os parâmetros reprodutivos podem ser satisfatórios para Bos taurus indicus, desde que criados em pastagem de boa qualidade e com suplementação mineral adequada.

Nos animais sexualmente imaturos, de acordo com classificação proposta por Vale Filho (1989), observaram-se diferenças $(\mathrm{P}<0,05)$ entre regimes alimentares para $\mathrm{PC}, \mathrm{CE}$ e Turb. O PC dos animais no $\mathrm{R} 3$ foi maior $(\mathrm{P}<0,05)$ que o dos animais do R2, e este foi maior do que o dos animais do R1. Da mesma forma, houve maior $\mathrm{CE}(\mathrm{P}<0,05)$, nos animais do $\mathrm{R} 3$ em comparação aos dos regimes R2 e R1, não foram observadas diferenças entre regimes alimentares nas demais características seminais Vol, Mot, Vigor, Conc, DM, Dm e DT.

O Turb que, em geral, não é muito alto, tem pouca importância para a seleção de touros jovens (Bos taurus indicus) aos dois anos de idade.

Tabela 3. Médias de peso corporal (PC), circunferência escrotal (CE), características seminais e classificação andrológica por pontos (CAP) em touros Tabapuã com dois anos de idade, classificados como sexualmente maturos, de acordo, com o regime alimentar

\begin{tabular}{cccccccccccc}
\hline $\begin{array}{c}\text { Regime } \\
\text { alimentar }\end{array}$ & $\begin{array}{c}\mathrm{PC} \\
(\mathrm{kg})\end{array}$ & $\begin{array}{c}\mathrm{CE} \\
(\mathrm{cm})\end{array}$ & $\begin{array}{c}\text { Vol } \\
(\mathrm{ml})\end{array}$ & $\begin{array}{c}\text { Conc. } \\
\left(\mathrm{x} 10^{6} / \mathrm{ml}\right)\end{array}$ & $\begin{array}{c}\text { Turb. } \\
(1-5)\end{array}$ & $\begin{array}{c}\text { Mot. } \\
(\%)\end{array}$ & $\begin{array}{c}\text { Vigor } \\
(1-5)\end{array}$ & $\begin{array}{c}\text { DM } \\
(\%)\end{array}$ & $\begin{array}{c}\text { Dm } \\
(\%)\end{array}$ & $\begin{array}{c}\text { DT } \\
(\%)\end{array}$ & $\begin{array}{c}\text { CAP } \\
(0-100)\end{array}$ \\
\hline 1 & $388,53 \mathrm{c}$ & $30,81 \mathrm{c}$ & $2,92 \mathrm{c}$ & 329,16 & 2,18 & 54,24 & 4,94 & 9,45 & 4,33 & 13,79 & 69,91 \\
2 & $412,32 \mathrm{~b}$ & $31,56 \mathrm{~b}$ & $3,93 \mathrm{~b}$ & 337,62 & 2,60 & 57,61 & 4,80 & 11,40 & 5,34 & 17,94 & 71,17 \\
3 & $497,15 \mathrm{a}$ & $33,81 \mathrm{a}$ & $4,83 \mathrm{a}$ & 342,17 & 2,37 & 54,55 & 4,71 & 13,47 & 5,29 & 18,75 & 75,86 \\
\hline
\end{tabular}

Valores seguidos por letras distintas na mesma coluna diferem entre si pelo teste Student-Newman-Keuls $(\mathrm{P}<0,05)$.

$\mathrm{Vol}=$ volume seminal, Turb=turbilhonamento, $\mathrm{Mot}=$ motilidade, Conc=concentração espermática, DM=defeitos maiores,

$\mathrm{Dm}=$ defeitos menores, $\mathrm{DT}=$ defeitos totais, $\mathrm{CAP}=$ classificação andrológica por pontos (Vale Filho, 1989). Maturos

sexualmente $=\mathrm{DM}<15 \%$ e DT $<30 \%$ (Vale Filho et al., 1989).

$1=$ somente em pasto; $2=$ pasto+suplementação na seca; $3=$ estabulado. 
Tabela 4. Médias de peso corporal (PC), circunferência escrotal (CE) e características seminais em touros Tabapuã com dois anos de idade, classificados como imaturos sexualmente, de acordo com regime alimentar

\begin{tabular}{|c|c|c|c|c|c|c|c|c|c|c|}
\hline $\begin{array}{l}\text { Regime } \\
\text { alimentar }\end{array}$ & $\begin{array}{l}\mathrm{PC} \\
(\mathrm{kg})\end{array}$ & $\begin{array}{c}\mathrm{CE} \\
(\mathrm{cm})\end{array}$ & $\begin{array}{l}\text { Vol } \\
(\mathrm{ml})\end{array}$ & $\begin{array}{l}\text { Turb. } \\
(1-5)\end{array}$ & $\begin{array}{l}\text { Mot. } \\
(\%)\end{array}$ & $\begin{array}{l}\text { Vigor } \\
(1-5)\end{array}$ & $\begin{array}{c}\text { Conc. } \\
\left(\times 10^{6} / \mathrm{ml}\right)\end{array}$ & $\begin{array}{l}\mathrm{DM} \\
(\%)\end{array}$ & $\begin{array}{l}\mathrm{Dm} \\
(\%)\end{array}$ & $\begin{array}{l}\text { DT } \\
(\%)\end{array}$ \\
\hline 1 & $367,36 \mathrm{c}$ & $29,56 b$ & 2,68 & $1,07 b$ & 40,55 & 4,59 & 319,73 & 38,40 & 9,00 & 45,92 \\
\hline 2 & $402,69 b$ & $29,55 b$ & 3,86 & $1,98 \mathrm{a}$ & 47,07 & 4,41 & 295,18 & 34,02 & 7,67 & 41,69 \\
\hline 3 & $479,22 \mathrm{a}$ & $32,96 \mathrm{a}$ & 3,24 & $1,78 \mathrm{a}$ & 43,16 & 4,58 & 403,84 & 23,28 & 7,89 & 31,17 \\
\hline
\end{tabular}

A CE dos animais sexualmente maturos foi maior do que a dos sexualmente imaturos (Tab. 3 e 4). Os animais maturos do R3 apresentaram maior $\mathrm{CE}(>30 \mathrm{~cm})$, em relação aos dos regimes R2 e R1, cujos DM e DT foram, respectivamente, $>15$ e $>30 \%$, que indicam a qualidade da gametogênese e evidenciam ausência de maturidade sexual (Tab. 4). Os valores dessas características (Tab. 3) são referentes a animais sexualmente maturos, aptos para a reprodução e capazes de obterem bons índices de fecundação (Vale Filho et al., 1989).
As porcentagens de touros sexualmente maturos, imaturos, e com $\mathrm{CAP}>60$ e $\mathrm{CAP}<60$ pontos, aos dois anos de idade, criados sob os três regimes nutricionais, são apresentados na Tab. 5. De modo geral, não houve efeito do regime alimentar sobre a maturidade e a CAP. Isso indica que animais criados em boa pastagem de Brachiaria brizantha e com suplementação mineral balanceada podem expressar a maturidade sexual e estarem aptos para a reprodução (CAP>60) aos dois anos de idade.

Tabela 5. Médias das porcentagens de touros Tabapuã, sexualmente maturos e imaturos, bem como as porcentagens do número de touros com $\mathrm{CAP}>60$ e $\mathrm{CAP}<60$, aos dois anos de idade, criados sob três regimes alimentares

\begin{tabular}{ccccc}
\hline Regimealimentar & Maturo (\%) & Imaturo (\%) & CAP $>60(\%)$ & CAP $<60(\%)$ \\
\hline 1 & $55,00 \mathrm{a}$ & $45,00 \mathrm{a}$ & $46,67 \mathrm{a}$ & $53,33 \mathrm{a}$ \\
2 & $60,27 \mathrm{a}$ & $39,73 \mathrm{a}$ & $50,68 \mathrm{a}$ & $49,32 \mathrm{a}$ \\
3 & $70,31 \mathrm{a}$ & $29,69 \mathrm{a}$ & $62,50 \mathrm{a}$ & $37,50 \mathrm{a}$ \\
\hline
\end{tabular}

Letras iguais na mesma coluna, dentro de cada característica, não diferem estatisticamente entre si pelo Qui-quadrado ( $\mathrm{P}>0,05)$.

Maturos sexualmente: defeitos espermáticos maiores $<15 \%$ e defeitos espermáticos totais $<30 \%$ (Vale Filho et al., 1989).

$\mathrm{CAP}=$ classificação andrológica por pontos (Vale Filho, 1989)

$1=$ somente em pasto; 2 =pasto+suplementação na seca; $3=$ =stabulado.

\section{CONCLUSÕES}

Machos Tabapuã com um ano de idade, apresentando peso corporal e circunferência escrotal satisfatórios, são impróprios para a reprodução por serem sexualmente imaturos e apresentarem número elevado de espermatozóides no ejaculado com defeitos maiores. A suplementação alimentar influi positivamente no peso corporal, na circunferência escrotal e nas características físicas do sêmen e apresentam menor efeito sobre a maturidade e a classificação andrológica por pontos de touros jovens Tabapuã, aos dois anos de idade.

\section{REFERÊNCIAS BIBLIOGRÁFICAS}

BERGMANN, J. A. G. Melhoramento genético da eficiência reprodutiva em bovinos de corte. Rev. Bras. Reprod. Anim., v.1, supl., p.70-86, 1993.

BLOM, E. The ultrastructure of some characteristic sperm defects and a proposal for a new classification of the bull spermiogram. Nord. Vet. Med., v.25, p.383-391, 1973.

DIAS, J. C.; SALVADOR, D. F.; ANDRADE, V. J. et al. Perfil andrológico de tourinhos da raça Guzerá, submetidos a três manejos nutricionais. In: REUNIÃO DA SOCIEDADE BRASILEIRA DE ZOOTECNIA, 40., 2003, 
Santa Maria. Anais... Santa Maria: SBZ, 2003. CD-ROM.

DODE, M.A.N. A importância do exame andrológico na avaliação de touros. Campo Grande: Embrapa Gado de Corte, 1997 (Boletim Informativo, 10).

FONSECA, V. O. O touro no contexto da eficiência reprodutiva do rebanho. Inf. Agropec., v.21, p.48-63, 2000.

FONSECA, V. O.; SANTOS, N. R.; MALINSKI, P. R. Classificação andrológica de touros zebus (Bos taurus indicus) com base no perímetro escrotal e características morfo-físicas do sêmen. Rev. Bras. Reprod. Anim., v.21, p.3639, 1997.

HANCOCK, J. L. The morphology of boar spermatozoa. J. Roy. Micro. Soc., v.76, p.84-97, 1957.

MANUAL para exame andrológico e avaliação de sêmen animal. 2. ed. Belo Horizonte: CBRA., 1998. $49 \mathrm{p}$.

REKWOT, P. I.; LAMIDI, O. S.; ADAMU, A. $M$. et al. Reproductive performance of Bunaji bulls grazing natural pasture and receiving supplements containing palm kernel meal. Nig. Vet. J., v.18, p.26-36, 1997 b.

RODRIGUEZ-URBINA, M. A.; GOICOCHEALLAQUE, J.; MADRID-BURY, N. et al. Comportamiento reproductivo de toretes postpuberales 5/8 Brahman suplementados con yacija. Rev. Cient. Fac. Cienc. Vet. Univ. Zulia, v.12, supl. 2, p.434-436, 2002.

SILVA, A. E. D. F.; DODE, M. A.; PORTO, J. A.; et al. Estacionalidade na atividade sexual de machos bovinos Nelore e mestiços Fleckvieh e ChianinaxNelore: características biométricas testiculares. Pesq. Agropec. Bras., v.26, p.1745$1750,1991$.

SISTEMA de análises estatísticas e genéticas SAEG. Versão 9.0. Viçosa, MG: UFV, 2004.

TEGEGNE, A.; ENTWISTLE, K. W.; MUKASA MUGERWA, E. Gonadal and extragonadal sperm reserves and testicular histometric characteristics in zebu and crossbred bulls: effect of dry season nutritional supplementation. Anim. Reprod. Sci., v.29, p.2533, 1992a.

TEGEGNE, A.; ENTWISTLE, K. W.; MUKASA MUGERWA, E. Nutritional influences on growth and onset of puberty in Boran and Boran x Friesian bulls in Ethiopia. Theriogenology, v.37, p.1005-1016, 1992b.

UNANIAN, M. M.; SILVA, A. E. D. F.; McMANUS, C.; et al. Características biométricas testiculares para avaliação de touros zebuínos da raça Nelore. Rev. Bras. Zootec., v.29, p.136-144, 2000.

VALE FILHO, V. R. Padrões de sêmen bovino, para o Brasil: Análise e sugestões. In: CONGRESSO BRASILEIRO DE REPRODUÇÃO ANIMAL, 8., 1989, Belo Horizonte. Anais... Belo Horizonte: CBRA, 1989. p. 94-118.

VALE FILHO, V. R. Testicular growth and breeding soundness of chimeric bulls. 1986. 183f. Tese (Doutorado) - University of Guelph, Canadá.

VALE FILHO, V.R. Subfertilidade em touros: parâmetros para avaliação andrológica e conceituação geral. Cad. Téc. Vet. Zootec., n.35, p.81-87, 2001.

VALE FILHO, V.R.; ANDRADE, V.J.; SALVADOR, D.F. Melhoramento genético e manejo reprodutivo de touros jovens Tabapuã. Rev. Bras. Zebu - ABCZ, n.12, p.44-47, 2003.

VALE FILHO, V.R.; REIS, S. R.; PEREIRA, J. C. C. et al. Efeitos do ambiente na maturação sexual de touros Nelore aos 24 meses de idade. Rev. Bras. Reprod. Anim., v. supl. 1, p.203-204, 1989.

VALVASORI, E.; TROVO, J. B. F.; PROCKNOR, M.; et al. Biometria testicular em tourinhos Gir, Guzerá, Nelore e Caracu. Bol. Industr. Anim., v.42, p.155-166, 1985. 